\title{
Sensor-based Dynamic Trajectory Planning for Smooth Door Passing of Intelligent Wheelchairs
}

\author{
Sen Wang, Ling Chen, Huosheng $\mathrm{Hu}$ and Klaus McDonald-Maier
}

\begin{abstract}
Traditionally, it is difficult for elderly and disabled people to control a wheelchair passing a narrow doorway manually. This paper presents a dynamic trajectory planning algorithm for wheelchairs to pass a door smoothly and automatically. It is a sensor-based approach in which two laser rangefinders are deployed in the wheelchair for real-time door detection. To generate smooth trajectories that enable a wheelchair to pass a door perpendicularly, Bézier curve based trajectories are calculated repeatedly during the whole course of door passing. The proposed approach is tested on a real wheelchair and the experimental results are presented to show the good performance and effectiveness of our proposed automatic door passing strategy.
\end{abstract}

\section{INTRODUCTION}

Door passing is considered as the fundamental capability of intelligent motorized wheelchairs that are operated in an indoor environment. The wheelchair users are normally elderly and disabled, who may suffer some types of disabilities such as Parkinson's disease. It is difficult for them to operate wheelchair using a traditional joystick to pass through the constrained doorway. Therefore, there is a great demand for intelligent wheelchairs that are able to provide automatic door passing functionality so that wheelchair users can easily travel through confined and narrow doorways without carer supervision.

The door passing problem can be addressed by two stages, namely trajectory planning [1], [2] and trajectory tracking [3], [4]. Up to now, many approaches have been worked on finding effective solutions to this problem. For instance, a frontier point method integrated with simultaneous localization and mapping was used for door passing of a wheelchair in [5]. However, it needs to continuously generate the mean frontier points, which makes the curves not smooth and time consuming. A door passage mode was used in the TAO wheelchair to achieve automatic door passing[6]. In general, most of existing intelligent wheelchairs can only pass a doorway very slowly and the curvature of the door passing trajectory is not smooth or continuous [6].

Gulati et al.[7] proposed a B-spline based graceful motion control for an intelligent wheelchair, enabling the wheelchair to pass through a narrow doorway. However, their implementation is based on a simulated wheelchair kinematic model and no real wheelchair is utilized. In addition, they assumed the wheelchair has a local map available to it, which is not necessarily feasible in many real-world applications. In [8], a Bézier curve based method, where the trajectory is

The authors are with School of Computer Science and Electronic Engineering, University of Essex, Colchester $\mathrm{CO} 43 \mathrm{SQ}$, United Kingdom swangi, lcheno, hhu, kdm at essex.ac.uk produced only once during the whole period of door passing, is proposed, which suffers from the accumulated errors of odometry and further causes inaccuracy of door passing. The implementation of the door passing algorithm is presented in [9] by using a Pioneer robot as a semi-simulation, which does not take the practical problems into account.

In order to pass through the narrow doorway smoothly and in real time using a real wheelchair, this paper proposes an effective strategy by refining the desired trajectory dynamically. It is a sensor-based approach in which two laser scanners are deployed for dynamic detection of a door frame. This paper also presents the new concept of the operation point and the desired position in the implementation of the dynamic trajectory planning for real-time performance.

The rest of this paper is organized as follows. Section II gives a brief problem description. In Section III, after designing the door passing strategy, several measures for its implementation are elaborated. Section IV presents the experimental results using a real wheelchair which verify the feasibility of our method in practice. Finally, a brief conclusion and future work are presented in Section V.

\section{PROBLEM DESCRIPTION}

The door passing problem of a wheelchair can be considered as a trajectory planning problem. The purpose of our proposed algorithm is to first generate an optimized smooth trajectory, then to control the wheelchair to track it. In this way, as long as the desired trajectory which passes through the doorway is well generated, and the wheelchair is able to track it accurately, the door passing problem will be achieved successfully. In practice, we only need to ensure one specific position on the wheelchair, which is defined as the operation point in this paper, to track its desired trajectory.

Fig.1 shows the schematic description of the door passing process of a wheelchair. The wheelchair in the corridor intends to traverse the door and enter the room. To generate a smooth door passing trajectory for the wheelchair, its geometrical feature and size should be taken into consideration. In this paper, either the position of laser $P_{s}^{l}$ (green square) or the center of the rear wheels $P_{s}^{c}$ (red circle) can be selected as the operation point. Thus, there are two possible local frames which are represented as $\{\mathrm{W}, \mathrm{L}\}$ (green dashed line) and $\{\mathrm{W}, \mathrm{C}\}$ (red solid line), whose origins are $P_{s}^{l}$ and $P_{s}^{c}$ respectively. $P_{d}$ is the middle position of the door in the global frame $\{\mathrm{G}\} . P_{s}\left(P_{s}^{l}\right.$ and $\left.P_{s}^{c}\right)$ and $P_{d}\left(P_{d}^{o}\right.$ and $\left.P_{d}^{o s}\right)$ are current and desired positions of the wheelchair respectively, and their corresponding headings are $H_{s}$ and $H_{d}$. Unless otherwise stated, all the positions and orientations throughout 


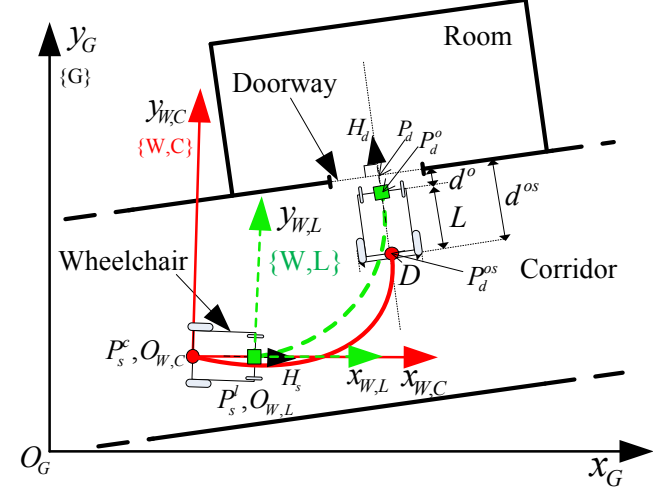

Fig. 1. The schematic description of door passing process of a wheelchair.

this paper are based on the frame $\{\mathrm{W}, \mathrm{C}\}$. The reason will be given in Section III-B.2.

To enable a wheelchair to successfully pass a narrow doorway, the door passing strategy should comply with the following criteria:

- The actual trajectory of the wheelchair should be smooth without oscillating or ambiguity.

- The curvature of the trajectory should be limited to a certain range for safety due to the kinematical constraint of the wheelchair.

- The heading of the wheelchair should be perpendicular to the door plane when the wheelchair arrives at $P_{d}$.

\section{Door PASSing StRATEgy and IMPLEMENTATION}

In this section, the door passing strategy and several techniques for implementation are presented. Several key problems are considered in practice so that the wheelchair can successfully pass a narrow doorway.

\section{A. Door Passing Strategy}

This door passing strategy includes door finding, optimization based trajectory planning and wheelchair control. Because it dynamically generates a smooth trajectory whenever the door is detected, the door passing can be performed without the odometry. This can greatly reduce the various disturbances from a real wheelchair and uncertain environment, making the door passing safe and effective.

1) Door Finding: The purpose of door finding is to obtain the coordinates $P_{d}$ of the middle position of the door and the slope $K_{w}$ of the wall. $P_{d}$ and $K_{w}$ will then be used to generate a Bézier curve in III-A.2. Segmentation and Line Fitting are the main procedures applied to calculate $P_{d}$ and $K_{w}$.

a) Segmentation: The purpose of segmentation is to divide one scan of laser data points into several different subsets so that the points of each subset belong to a straight line. First, an Adaptive Breakpoint Detector (ABD)[10] is adopted to find continuous point groups by detecting breakpoints where some kind of discontinuity has occurred. Then, Iterative End-Point Fit algorithm (IEPF)[11] is applied to separate each group into different subsets so that points of each subset belong to the same straight line. For every two subsets, we calculate the distance $D_{r f}$ between the rear end point $\left(P_{r}\right)$ of the first subset and the front end point $\left(P_{f}\right)$ of the second subset. If $\left|D_{r f}-W_{d}\right|<D_{t h}$ where $W_{d}$ is the door width and $D_{t h}$ is threshold of the absolute substraction, then the door is found and $P_{d}=\left(P_{r}+P_{f}\right) / 2$.

b) Line Fitting: The calculation of $K_{w}$ can be realized by fitting the straight line composed by the laser data points of the wall. Line fitting means determining line parameters. Suppose that there are a set of points $R=\left\{\left(x_{i}, y_{i}\right), i=\right.$ $1: k\}$ that are needed to be fitted to a specific line, whose slope and intercept are $k$ and $q$ respectively. Then these two parameters can be calculated by the Least Squares Fitting based line fitting algorithm which is represented as:

$$
\begin{gathered}
S_{x}=\sum_{i=1}^{k} x_{i} ; \quad S_{y}=\sum_{i=1}^{k} y_{i} ; \\
S_{x x}=\sum_{i=1}^{k} x_{i}^{2} ; \quad S_{y y}=\sum_{i=1}^{k} y_{i}^{2} ; \quad S_{x y}=\sum_{i=1}^{k} x_{i} y_{i} ; \\
k=\frac{-S_{x} S_{y y}+S_{y} S_{x y}}{S_{y} S_{x x}-S_{x} S_{x y}} ;
\end{gathered}
$$

where (1) provides the right solution of the slope of the fitted straight line.

2) Trajectory Planning based on Bézier Curve: After the door is detected by laser rangefinder (LRF), the Bézier curve based trajectory is continuously refined for the wheelchair to follow. A cubic Bézier curve can be denoted as

$$
\begin{aligned}
& x(t)=A_{0}(1-t)^{3}+3 A_{1}(1-t)^{2} t+3 A_{2}(1-t) t^{2}+A_{3} t^{3} \\
& y(t)=B_{0}(1-t)^{3}+3 B_{1}(1-t)^{2} t+3 B_{2}(1-t) t^{2}+B_{3} t^{3}
\end{aligned}
$$

where $P_{0}\left(A_{0}, B_{0}\right), P_{1}\left(A_{1}, B_{1}\right), P_{2}\left(A_{2}, B_{2}\right)$ and $P_{3}\left(A_{3}, B_{3}\right)$ are the control points of a Bézier curve.

In Fig.1, $P_{0}$ and $P_{3}$ are $P_{s}$ and $P_{d}$ respectively. Then, the control points $P_{1}$ and $P_{2}$ are found by the constrained optimization method such that the curvature of the Bézier curve and the rate of its change are as small as possible. According to (2), the curvature of a Bézier curve with respect to $t$ is

$$
\kappa(t)=\frac{\dot{x}(t) \ddot{y}(t)-\dot{y}(t) \ddot{x}(t)}{\left(\dot{x}^{2}(t)+\dot{y}^{2}(t)\right)^{3 / 2}} .
$$

Therefore, $P_{1}$ and $P_{2}$ can be computed by the following constrained optimization problem:

$$
\begin{array}{cc}
\min _{P_{1}, P_{2}} & \int_{0}^{1}\left[(\kappa(t))^{2}+(\dot{\kappa}(t))^{2}\right] d t \\
\text { s.t. } & \left\|P_{0}-P_{c}\right\|=\left\|P_{1}-P_{0}\right\|+\left\|P_{1}-P_{c}\right\| \\
& \left\|P_{3}-P_{c}\right\|=\left\|P_{2}-P_{3}\right\|+\left\|P_{2}-P_{c}\right\| \\
& \operatorname{sign}\left(\omega_{1}\right) \cdot \operatorname{sign}\left(\omega_{2}\right)>0
\end{array}
$$

where $\dot{\kappa}(t)$ is the first derivative of curvature; $P_{c}$ is the intersection point of lines $P_{0} P_{1}$ and $P_{2} P_{3}$ whose slopes are 0 and $-1 / K_{w} ;\|\cdot\|$ denotes the 2-norm; and $\operatorname{sign}\left(\omega_{i}\right), i=1,2$, are the rotation direction of the $\omega_{i}$ where

$$
\omega_{1}=\overrightarrow{P_{0} P_{1}} \times \overrightarrow{P_{1} P_{2}} \quad \omega_{2}=\overrightarrow{P_{1} P_{2}} \times \overrightarrow{P_{2} P_{3}} .
$$




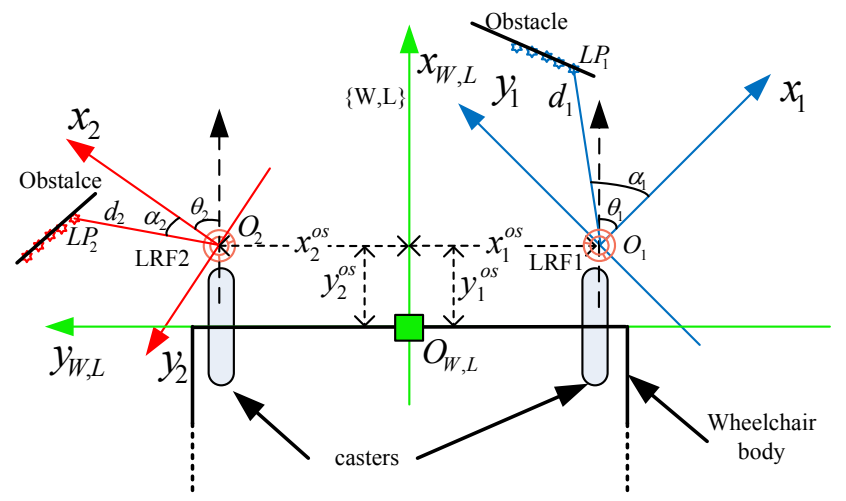

Fig. 2. The schematic description of converting scanned data of two LRFs to that of one virtual laser.

The constraints (4b) and (4c) are used to make sure that $P_{1}$ and $P_{2}$ are located on the tangent lines at positions $P_{0}$ and $P_{3}$ because the start (end) of the curve should be tangent to the first (last) section of the Bézier polygon. (4d) ensures that there is no sharp bend or sudden change of curvature with the completely convex feature of the generated curve[12]. Thus, the calculated $P_{1}$ and $P_{2}$ can meet the various requirements described in II and provide an optimal trajectory for the wheelchair to follow.

Whenever the door is found, the trajectory is updated, i.e., $P_{0}$ is set as $(0,0)$ and $P_{3}$ is calculated based on the measured door position. Once the trajectory is refined, the wheelchair is controlled to follow it using a PID controller.

\section{B. Implementation}

1) Laser Combination: Hokuyo's URG-04LX LRF is used as the range sensor for the wheelchair to perceive its surrounding environment, i.e., to find the middle position of the door. The detection angle range of a LRF is $240^{\circ}$, and the rest is a blind area where the laser is not able to scan. Therefore, it is necessary to combine two LRFs to cover the whole area of $360^{\circ}$. We mount two LRFs on the two sides of the wheelchair, and then integrate the two laser data to cover the surrounding of the wheelchair, i.e. forming a virtual laser.

As shown in Fig.2, the two LRFs, namely LRF1 and LRF2, are located in front of the wheelchair. Their coordinate frames are defined as $x_{1} O_{1} y_{1}$ (blue) and $x_{2} O_{2} y_{2}$ (red) respectively. The coordinate frame of the virtual laser is the local frame $\{\mathrm{W}, \mathrm{L}\}$ defined in Fig.1. Thus, combining two lasers is actually to convert scanned data of two LRFs to the coordinate frame $\{\mathrm{W}, \mathrm{L}\}$ of the virtual laser. Rotation and translation are used to do the conversion. The offset angles between $x_{1} O_{1} y_{1}$ and $\{\mathrm{W}, \mathrm{L}\}, x_{2} O_{2} y_{2}$ and $\{\mathrm{W}, \mathrm{L}\}$ are defined as $\theta_{1}$ and $\theta_{2}$ respectively, which will be used for rotation.

The offset coordinates of the origins of the two frames $x_{1} O_{1} y_{1}$ and $x_{2} O_{2} y_{2}$ in frame $\{\mathrm{W}, \mathrm{L}\}$ are $\left(x_{1}^{o s}, y_{1}^{o s}\right)$ and $\left(x_{2}^{o s}, y_{2}^{o s}\right)$ respectively, which will be used for translation. The polar coordinate of any scanned data point $L P 1$ of LRF1 is $\left(\alpha_{1}, d_{1}\right)$ in frame $x_{1} O_{1} y_{1}$. Therefore, its corresponding

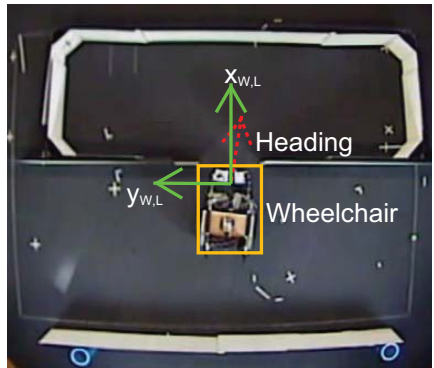

(a) Position of wheelchair

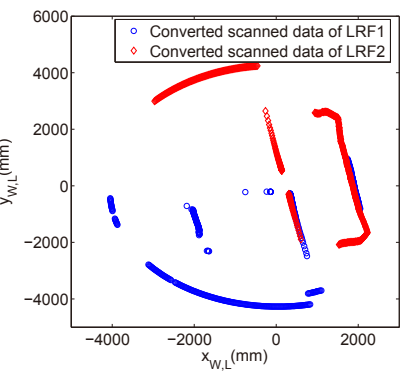

(b) Converted scanned data
Fig. 3. Validity checking of the laser combination formulation. (a) The position of the wheelchair where two LRFs scan the environment; (b) Converted scanned data of each LRF

coordinates in frame $\{\mathrm{W}, \mathrm{L}\}$ can be calculated as

$$
\begin{gathered}
x_{L P 1}=d_{1} \cos \left(\alpha_{1}-\theta_{1}\right)+x_{1}^{o s} \\
y_{L P 1}=d_{1} \sin \left(\alpha_{1}-\theta_{1}\right)+y_{1}^{o s} \\
\alpha_{L P 1}=\arctan 2\left(y_{L P 1}, x_{L P 1}\right) \\
d_{L P 1}=\sqrt{x_{L P 1}^{2}+y_{L P 1}^{2}}
\end{gathered}
$$

where $\left(\alpha_{L P 1}, d_{L P 1}\right)$ is the polar coordinate of $L P 1$ in frame $\{\mathrm{W}, \mathrm{L}\}$. The similar formulations can also apply to any scanned data point of LRF2 in frame $x_{2} \mathrm{O}_{2} y_{2}$. Thus, the combined lasers can be seen as a virtual laser positioned in the middle of the wheelchair front as represented as $O_{W, L}$ in Fig.2.

In order to verify the validity of two laser combination (6), one scan of two LRFs is recorded and the scanned data is converted into coordinate frame $\{\mathrm{W}, \mathrm{L}\}$ as shown in Fig.3, where Fig.3a shows the wheelchair position where the scanning occurs, and Fig. $3 \mathrm{~b}$ is the result of the conversion of scanned data into frame $\{\mathrm{W}, \mathrm{L}\}$. As can be seen in Fig.3b, both the slope and the position of the wall line accurately match with the situation shown in Fig.3a, and the overlapped part of the scanned data from the two LRFs almost has the same coordinates in their common frame $\{\mathrm{W}, \mathrm{L}\}$. These two aspects prove the validity of the laser combination.

2) Bézier Curve Generation: Although the strategy of the Bézier curve based trajectory generation has been designed above, it cannot be directly used for the wheelchair in practice, especially under the serious impact of the large size of the wheelchair. This section addresses this issue by determining the appropriate starting and ending points $P_{0}$ and $P_{3}$, which are also considered as the operation point $P_{s}$ and the desired position $P_{d}$ respectively. The refined strategy could generate the satisfied trajectories in reality, which are smooth and also suitable for the real wheelchair to follow.

a) Selection of an operation point $P_{s}$ : When producing a new Bézier curve, our method always refines the coordinates of the control point $P_{0}$ to be $(0,0)$. However, as shown in Fig.1, the position of $P_{0}$ can be $P_{s}^{c}$ or $P_{s}^{l}$. Generally, $P_{s}^{l}$ should be chosen since all the door detection and measurements are based on the position of the laser. This is reasonable if the vehicle can be considered as a 


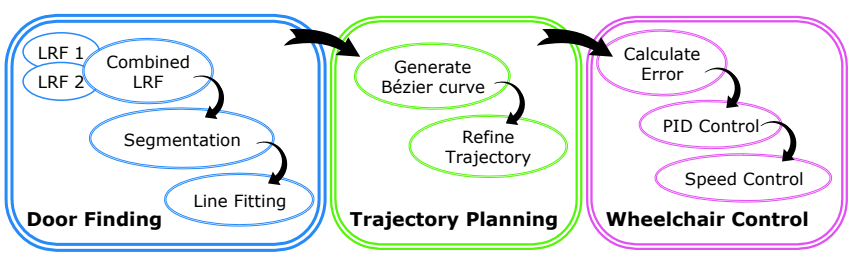

Fig. 4. Strategy architecture of door passing.

particle or the distance between the laser and the center of the driving wheels is small. Unfortunately, these two scenarios are unsuitable for the real wheelchair due to its large size. Moreover, it is difficult to control the rear wheels to make the front laser point follow the trajectory. Therefore, $P_{s}^{c}$ is selected as the control point $P_{0}$ when designing a Bézier curve based trajectory, which is the (red) solid line in Fig.1. Accordingly, the whole door passing strategy is based on the frame $\{\mathrm{W}, \mathrm{C}\}$. Meanwhile, this choice of $P_{s}^{c}$ is more robust to the errors of the wheelchair control than that of $P_{s}^{l}$ because the same error will cause more deflection for $P_{s}^{l}$ than $P_{s}^{c}$.

Because $P_{s}^{c}$ is far from $P_{s}^{l}$, the coordinates of the door calculated in the frame $\{\mathrm{W}, \mathrm{L}\}$ should be translated into the frame $\{\mathrm{W}, \mathrm{C}\}$. This translation is similar to the one used in III-B.1. However, it only has an offset along the $X$ axis and the length is the distance between $P_{s}^{c}$ and $P_{s}^{l}$, i.e., $L$ in Fig.1.

b) Selection of a desired position $P_{d}$ : When the wheelchair approaches to the middle of the door, the lasers may be collinear with the wall. Then they cannot detect the position of the door accurately or even cannot at all. Meanwhile, because of various disturbances in practice, the heading of the wheelchair cannot fully reach the expected one. Therefore, the wheelchair needs to adjust the heading to be perpendicular to the wall when passing the doorway in order to decrease the probability of colliding to the door frame.

In order to overcome this problem, an offset against the middle of the door is set when determining $P_{d}$ for the new trajectory so that there is enough front distance for lasers to detect the wall. In this way, the wheelchair can adjust its heading to be perpendicular to the door at the position $P_{d}^{o s}$ rather than $P_{d}$, see Fig.1. When the coordinates of the middle of the door $P_{d}$ and the desired heading of the wheelchair $H_{d}$ in the frame $\{\mathrm{W}, \mathrm{C}\}$ are obtained by $K_{w}, P_{d}^{o}$ whose offset to $P_{d}$ is $d^{o}$ can be calculated. However, because the operation point is $P_{s}^{c}$, the offset point $P_{d}^{o}$ should be further translated to the point $P_{d}^{o s}$, which can be used as the control point $P_{3}$ to generate the Bézier curve. $P_{d}^{o s}$ can be obtained by:

$$
\begin{aligned}
& \theta_{d}^{o s}=\arctan H_{d}, \quad \theta_{d}^{o s} \in\left(-90^{\circ}, 90^{\circ}\right) \\
& x_{d}^{o s}=d^{o s} \cos \theta_{d}^{o s}+x_{P_{d}} \\
& y_{d}^{o s}=d^{o s} \sin \theta_{d}^{o s}+y_{P_{d}}
\end{aligned}
$$

where $x_{d}^{o s}$ and $y_{d}^{o s}$ are the coordinates of $P_{d}^{o s}$, and $d^{o s}$ is the length of the offset including the real offset $d^{o}$ and the length of the wheelchair $L$.

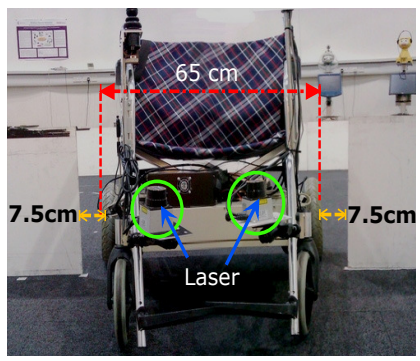

(a) Elevation of the wheelchair

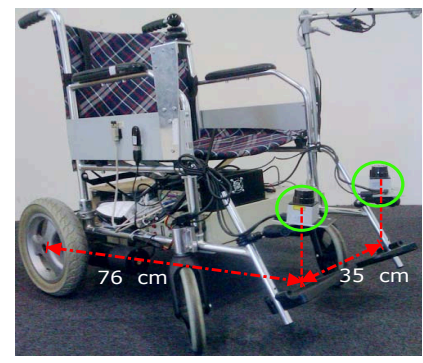

(b) Side elevation of the wheelchair
Fig. 5. Structure of the wheelchair and the lasers

\section{Strategy Architecture}

The proposed door passing strategy can be seen in Fig.4. There are three main modules, namely door finding, trajectory planning and wheelchair control. In door finding module, the data from two lasers is combined together to find the door position by conducting segmentation and line fitting. Once the door position is found accurately, it is used by the trajectory planning module to generate desired Bézier curve based trajectory. Then in the wheelchair control module, the error between the desired trajectory and the wheelchair's current position is fed into a PID controller to calculate the velocities of two wheels so that the wheelchair can follow the desired trajectory. This process is executed repeatedly in real time.

\section{EXPERIMENTAL RESULTS}

In order to verify and evaluate the proposed method, a real wheelchair is used. As shown in Fig.5 and Fig.6, the length and width of the wheelchair are $85 \mathrm{~cm}$ and $65 \mathrm{~cm}$ respectively. The offset between the lasers and the rear wheels is $76 \mathrm{~cm}$, and the corresponding offset angles $\theta_{1}$ and $\theta_{2}$ are $46.36^{\circ}$ and $-56.50^{\circ}$ respectively. Since the width of the door is $80 \mathrm{~cm}$, the free space of the doorway for the wheelchair is only $7.5 \mathrm{~cm}$ on each side.

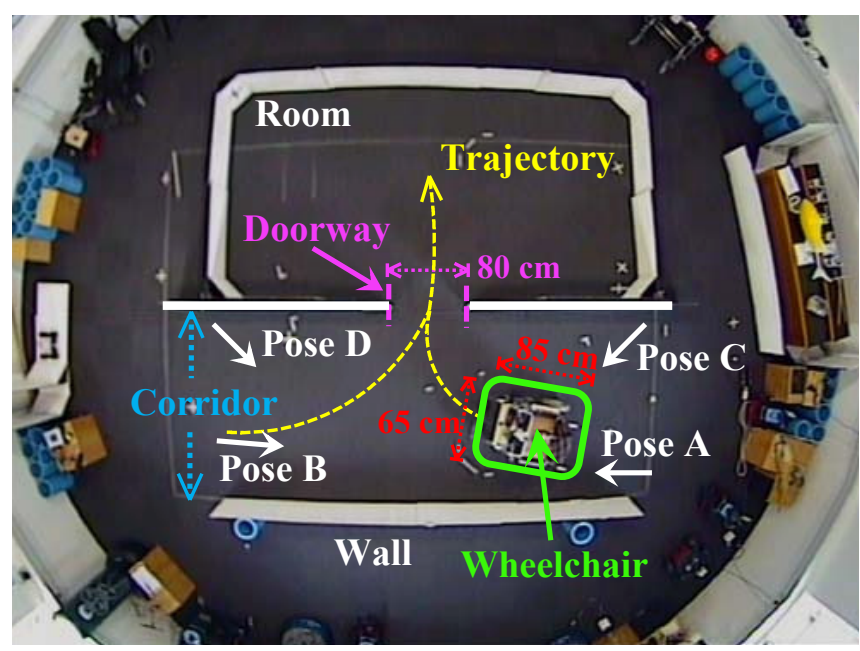

Fig. 6. Experimental scenario. 


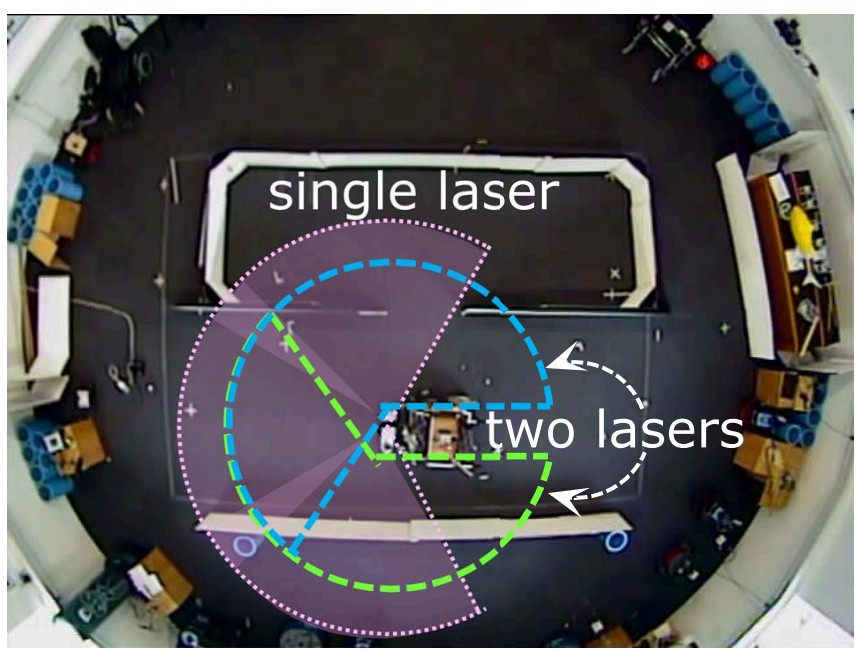

Fig. 7. Advantage of two lasers compared with single laser.

The experiment scenario is described in Fig.6, where there are several walls and a room. The wheelchair enclosed by the (green) solid line starts twice from each of the poses $A$, $B, C$ and $D$ to pass the doorway and enter the room. The ground truth information of the global coordinates of the wheelchair is obtained by the VICON system for evaluating our strategy.

It can be seen from Fig.7 that the single LRF cannot detect the whole frame of the door any more due to the big size of the wheelchair, which produces the wrong trajectory and inappropriate control. However, our two LRFs combination method can successfully address this problem and provide the full scope of scanning for door finding.

Fig. 8 shows the wheelchair trajectories for the four starting poses. For each starting pose, there are two trials conducted. It is obvious that no matter which starting pose the wheelchair is at, it is able to find and pass the door both smoothly and accurately without bumping into the door edges, which verifies the high accuracy and effectiveness of our proposed door passing strategy.

Fig.9 shows the actual wheelchair poses when arriving at the desired pose with different starting poses $A, B, C$ and $D$. As can be seen, almost all the actual positions lie within the neighboring area of the desired pose with certain errors, and most of the headings are perpendicular to the door line as expected, though some of them are slightly away from the desired one. There are mainly two reasons for these errors. First, the front castors of the wheelchair produce big influences on the orientations, which can be seen in our attached video ${ }^{1}$. In addition, the long offset produced by selecting the desired point $P_{d}$ magnifies the error of door finding. However, the horizontal offsets between the actual positions and the desired position are all still less than $7.5 \mathrm{~cm}$, which indicates that our strategy can reduce the impact of the practical disturbances and uncertainties for the wheelchair to pass the doorway successfully even with narrow free space.

\footnotetext{
${ }^{1}$ This video can also be found using this link: www.youtube.com/watch? $v=$ ahwG6CnqtmM $\&$ feature $=$ plcp
}

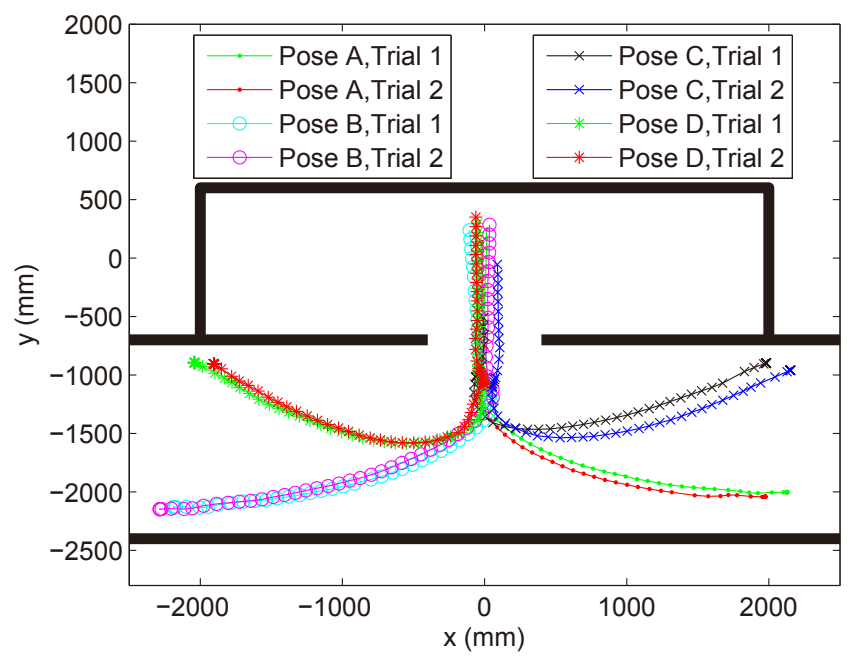

Fig. 8. Trajectories of the wheelchair.

Fig.10 presents the angular rates for each trial at three stages. In the first stage, the angular rate of the wheelchair increases until it almost reaches the middle of the door so that it can follow the desired trajectory. In the second stage, the wheelchair adjusts its heading to ensure the heading of the wheelchair is perpendicular to the door. In the third stage, the wheelchair goes straight forward to the room. It should be noticed that in the first stage of all the trials, the angular rate is smoothly increasing without sharp changes, which again proves the achievement of smooth door passing. In addition, for both trials of the same starting pose, the angular rates of the wheelchair overlap with each other, which indicates the high repeatability of our proposed door passing implementation strategy.

The snapshots of the trajectories starting from four poses $A, B, C$ and $D$ are presented in Fig.11, including several important poses, such as the starting pose, the poses before

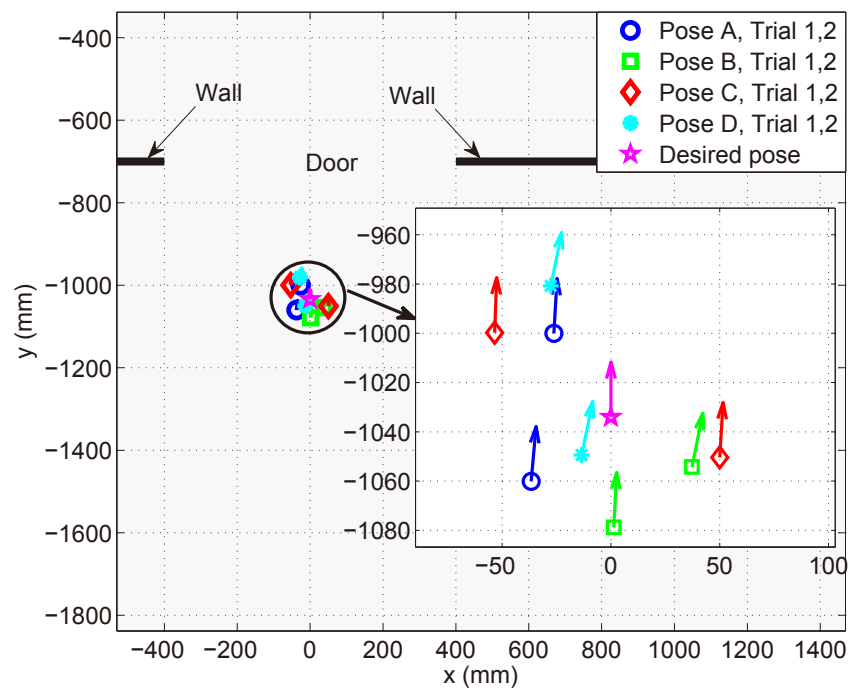

Fig. 9. Actual wheelchair poses when arriving at the desired pose with different starting poses $\mathrm{A}, \mathrm{B}, \mathrm{C}$ and $\mathrm{D}$. 


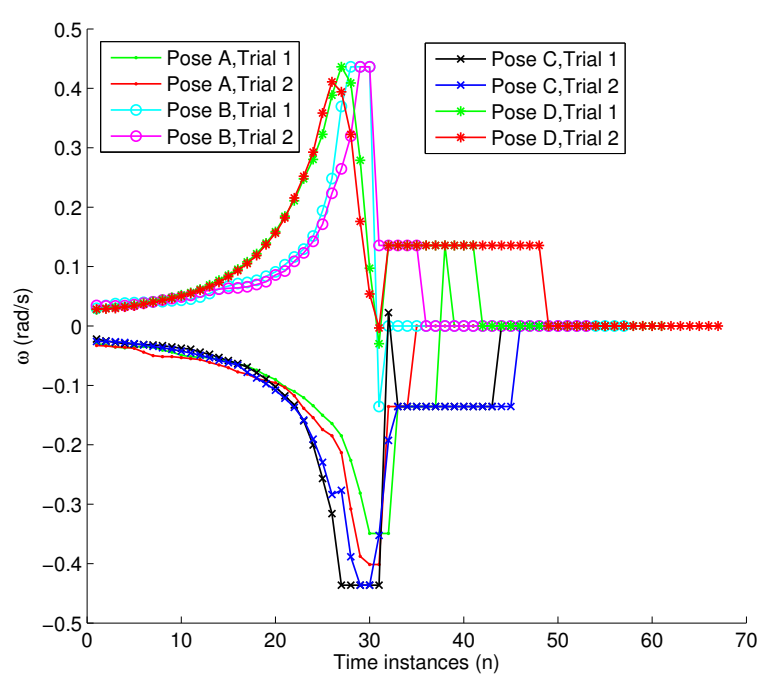

Fig. 10. Angular rate of the wheelchair.

and after passing the doorway. In all the fourth snapshots, the wheelchair is almost in the middle line of the door with the nearly perpendicular headings, which verifies the effectiveness of our proposed method. The fifth snapshots also demonstrate that the wheelchair can pass the doorway with the expected heading.

\section{CONCLUSIONS}

This paper focuses on solving the door passing problem for an intelligent wheelchair both theoretically and practically. A general door passing strategy is firstly presented in theory including door finding, optimization based trajectory planing and wheelchair control. Due to the large size of the wheelchair, several practical issues appear. Therefore, we further present various effective measures to enable the wheelchair to pass the doorway smoothly. These measures include combining two lasers, selecting the operation point and the desired position. The experiments of door passing are conducted with four different starting poses, each of which has 2 trials. The experimental results verify the effectiveness of our proposed door passing implementation strategy. Future work will focus on autonomous navigation of the wheelchair using the proposed door passing strategy.

\section{ACKNOWLEDGMENTS}

This research is financially supported by the EU COALAS project. The COALAS project has been selected in the context of the INTERREG IVA France (Channel) England European cross-border co-operation programme, which is cofinanced by the ERDF. Sen Wang and Ling Chen have been financially supported by scholarships from China Scholarship Council and University of Essex. Our thanks also go to Robin Dowling for his technical support during the research.

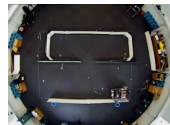

(a) Pose A 1

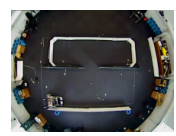

(f) Pose B 1

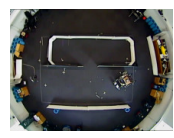

(k) Pose C 1

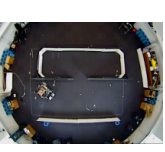

(p) Pose D

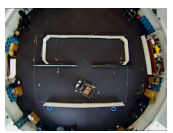

(b) Pose A 2
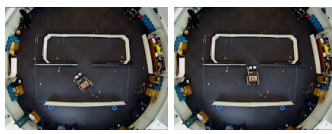

(d) Pose A 4

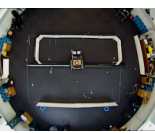

(e) Pose A 5
Fig. 11. Snapshots of the wheelchair's trajectories when passing doorway, starting from pose A, B, C and D. (a)-(e), (f)-(j), (k)-(o) and (p)-(t) are with Pose A, B, C and D respectively.

\section{REFERENCES}

[1] A. Scheuer and T. Fraichard, "Continuous-curvature path planning for car-like vehicles," in Proceedings of the 1997 IEEE/RSJ International Conference on Intelligent Robots and Systems, 1997., vol. 2. IEEE, 1997, pp. 997-1003.

[2] J. Choi, R. Curry, and G. Elkaim, "Path planning based on bézier curve for autonomous ground vehicles," in Advances in Electrical and Electronics Engineering-IAENG Special Edition of the World Congress on Engineering and Computer Science. IEEE, 2008, pp. 158-166.

[3] F. Martins, W. Celeste, R. Carelli, M. Sarcinelli-Filho, and T. BastosFilho, "An adaptive dynamic controller for autonomous mobile robot trajectory tracking," Control Engineering Practice, vol. 16, no. 11, pp. 1354-1363, 2008.

[4] J. Lee, C. Lin, H. Lim, and J. Lee, "Sliding mode control for trajectory tracking of mobile robot in the rfid sensor space," International Journal of Control, Automation and Systems, vol. 7, no. 3, pp. 429435, 2009.

[5] F. Cheein, C. De La Cruz, T. Bastos, and R. Carelli, "Slam-based cross-a-door solution approach for a robotic wheelchair," Int $J$ Adv Robot Syst, vol. 7, no. 2, pp. 155-164, 2010.

[6] R. Simpson, "Smart wheelchairs: A literature review." J Rehabil Res Dev, vol. 42, no. 4, p. 423, 2005.

[7] S. Gulati and B. Kuipers, "High performance control for graceful motion of an intelligent wheelchair," in IEEE International Conference on Robotics and Automation, 2008. ICRA 2008. IEEE, 2008, pp. 3932-3938.

[8] L. Chen, S. Wang, H. Hu, and K. McDonald-Maier, "Bézier curve based trajectory planning for an intelligent wheelchair to pass a doorway," in 2012 United Kingdom Automatic Control Council International Conference on Control. IEEE, 2012, pp. 339-344.

[9] S. Wang, L. Chen, H. Hu, and K. McDonald-Maier, "Doorway passing of an intelligent wheelchair by dynamically generating bézier curve trajectory," in 2012 IEEE International Conference on Robotics and Biomimetics. IEEE, 2012, pp. 1206-1211.

[10] G. Borges and M. Aldon, "Line extraction in $2 d$ range images for mobile robotics," Journal of Intelligent and Robotic Systems, vol. 40, no. 3, pp. 267-297, 2004.

[11] R. Duda and P. Hart, "Pattern classification and scene analysis," A Wiley-Interscience Publication, New York: Wiley, 1973, vol. 1, 1973.

[12] P. Laurent, P. Sablonnière, and L. Schumaker, Curve and surface design: Saint-Malo 99, ser. Innovations in applied mathematics. Vanderbilt University Press, 2000. 